\title{
Bedeutung der Expositionswege auf Bioakkumulation und Wirkung in C. elegans
}

\author{
Wolfgang Ahlf • Anett Matthäi · Kirsten Offermann
}

Eingegangen: 11. März 2009/Akzeptiert: 29. April 2009/Online veröffentlicht: 15. Mai 2009

(C) Springer-Verlag 2009

\begin{abstract}
Zusammenfassung Die unterschiedlichen Aufnahmewege von Schadstoffen wurden differenziert, wobei besonders die Bedeutung der partikelgebundenen Stoffe untersucht wurde. Die biologische Verfügbarkeit von Cadmium wurde mithilfe der quantitativen mRNA-Analyse über die Expression des ,cadmium responsive gene“ $c d r$ - in Caenorhabditis elegans bestimmt. Vergleichbare Versuche wurden mit Fluoranthen als organischen Schadstoff durchgeführt. Das Gen cyp-35C1 in C. elegans diente dabei als ein empfindlicher Anzeiger des Entgiftungssystems für PAK. Im Vergleich der Exposition über das Medium, mit und ohne Bakterien als Nahrung, wurde gezeigt, dass der entscheidende Expositionsweg für Bioakkumulation und Wirkung über Nahrungspartikel erfolgte. Partikel mit unterschiedlichen funktionellen Gruppen erhöhten ebenfalls die Cadmiumanreicherung im Nematoden, doch weitaus geringer als durch Bakterien oder einer Kombination aus beiden Partikelarten. Die Genexpression als Reaktion auf die Fluoranthenexposition zeigte nach Überschreiten eines Schwellenwertes, dass die Reproduktionsleistung der Nematoden reduziert wurde. Die Bioverfügbarkeit von Fluoranthen über Nahrungspartikel führte schneller zur cyp-Genexpression. Die interne Verfügbarkeit hängt somit entscheidend vom Aufnahmeweg ab.
\end{abstract}

Schlüsselwörter C. elegans $\cdot$ Bioverfügbarkeit $\cdot \mathrm{Cd}$. Fluoranthen $\cdot$ Nahrungspartikel

Verantwortliche Herausgeber: Jan Schwarzbauer - Peter Heininger · Evelyn Claus

W. Ahlf $(\triangle) \cdot$ A. Matthäi $\cdot$ K. Offermann Institut für Umwelttechnik und Energiewirtschaft, TUHH, Eißendorfer Straße 40, 21073 Hamburg, Deutschland

E-Mail: ahlf@tu-harburg.de

\section{Importance of uptake routes on bioaccumulation} and effects in $C$. elegans

\begin{abstract}
Different uptake routes of contaminants were analysed with focus on particle-bound pollutants. Bioavailability of cadmium was determined with quantitative mRNA-analysis using the expression of the "cadmium responsive gene" cdr- in Caenorhabditis elegans. Comparable experiments were performed with fluoranthene as organic pollutant. The exposure in medium was compared with and without bacteria as food particles. Both bioaccumulation and toxic effects were most influenced via the food exposure. Inorganic particles increased also the uptake of cadmium, but significantly less than food or mixtures of food and inorganic particles. The exposure of fluoranthene reduced the reproduction of the nematodes, if a gene expression threshold was exceeded. However, bioavailabilty of fluoranthene via food particles caused a faster response of the cyp-expression. Thus means, the internal availability is crucial influenced by the uptake route.
\end{abstract}

Keywords C. elegans $\cdot$ Bioavailability $\cdot \mathrm{Cd}$.

Fluoranthene $\cdot$ Food particles

\section{Einleitung}

Der benthisch lebende Nematode Caenorhabditis elegans kann Schadstoffe sowohl durch die freie Wasserphase, das Porenwasser, aber auch durch die Ingestion kontaminierter Partikel oder Nahrung aufnehmen. Obwohl der Aufnahmeweg von Schadstoffen über die Nahrung allgemein akzeptiert ist, wird er häufig bei einer Risikobewertung nicht als relevant berücksichtigt (z.B. MERAG 2007). Dabei wird das Konzept des biologischen Liganden-Modells (BLM) 
verwendet, wonach zusätzliche Liganden als Konkurrenten in einer Lösung agieren und somit die biologische Verfügbarkeit herabgesetzt wird (DiToro et al. 2005). Dieser Ansatz sollte sowohl für ein Schwermetall als auch für einen lipophilen Schadstoff geprüft werden.

\section{Material und Methoden}

In den Versuchsansätzen wurden entweder Cadmium oder Fluoranthen in wässrigem Medium eingesetzt. In einem zweiten Ansatz wurden dann Partikel als zusätzliche Liganden zugegeben. Die Reaktion der Nematoden wurde mit Biomarkern der Genexpression quantitativ als mRNABildung gemessen (näheres siehe Offermann et al. 2009; Matthäi 2009).

\section{Ergebnisse und Diskussion}

Abbildung 1 zeigt die Reaktion auf unterschiedliche Cadmiumexposition. Die biologische Verfügbarkeit wurde mithilfe der quantitativen mRNA-Analyse der Expression des "cadmium responsive gene“ cdr- in Caeno-rhabditis elegans bestimmt. Im Vergleich von Medium mit und ohne Bakterien als Nahrung ist zu sehen, dass der entscheidende Expositionsweg für Bioverfügbarkeit und Wirkung über Nahrungspartikel erfolgte. Die Genexpression wurde etwa um den Faktor 100 durch Bakterien im Vergleich zur Exposition allein über die Wasserphase erhöht. Partikel mit unterschiedlichen funktionellen Gruppen erhöhten ebenfalls die

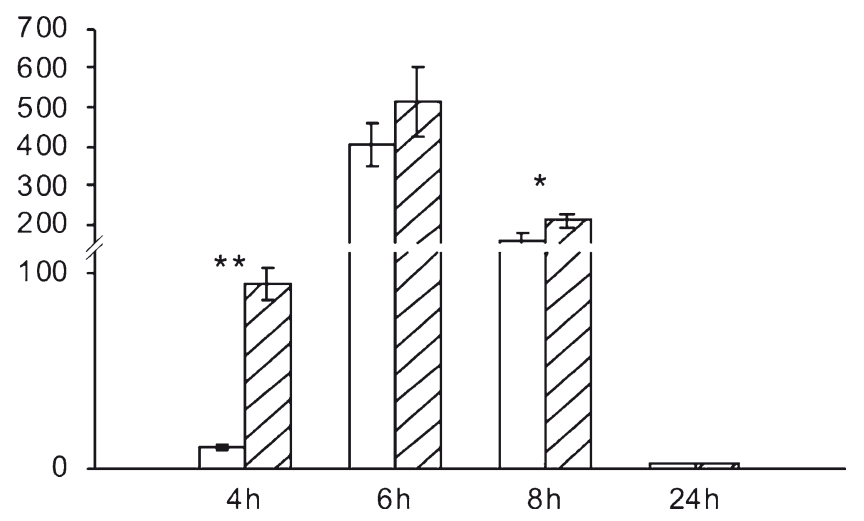

Abb. 2 Relative Expression von C. elegans cyp-35C1, induziert durch Inkubation in $0,9 \mathrm{mg} / \mathrm{L}$ Fluoranthen für 4-24 Stunden mit Bakterien (E. coli) (gestreifte Säulen) und ohne Bakterien (weiße Säulen)

Cadmiumverfügbarkeit im Nematoden, doch weitaus geringer als durch Bakterien oder einer Kombination aus beiden Partikelarten (Offermann et al. 2009).

Ähnliche Versuche wurden mit Fluoranthen (Flu) als organischen Schadstoff durchgeführt. Das Gen cyp-35C1 in C. elegans wurde als ein empfindlicher Anzeiger des Entgiftungssystems für PAK ausgewählt. Die Expression des Gens war konzentrationsabhängig zur Flu-Konzentration im wässrigen Medium bei einer Expositionsdauer von 6h. Diese Expositionszeit erzeugte keine irreversiblen Effekte, gemessen als Wachstum und Reproduktionsleistung (Matthäi 2009).

Bei einer Konzentration von $0,9 \mathrm{mg} / \mathrm{L}$ Flu wurden zeitund expositionsabhängige Effekte gemessen. Die Induktion
Abb. 1 Relative cdr-Genexpression nach Zugabe von Bakterien als Nahrungspartikel sowie Latexkugeln mit einer Belegung durch Carboxylgruppen sowie einer 1:1 Mischung (vgl. Offermann et al. 2009)

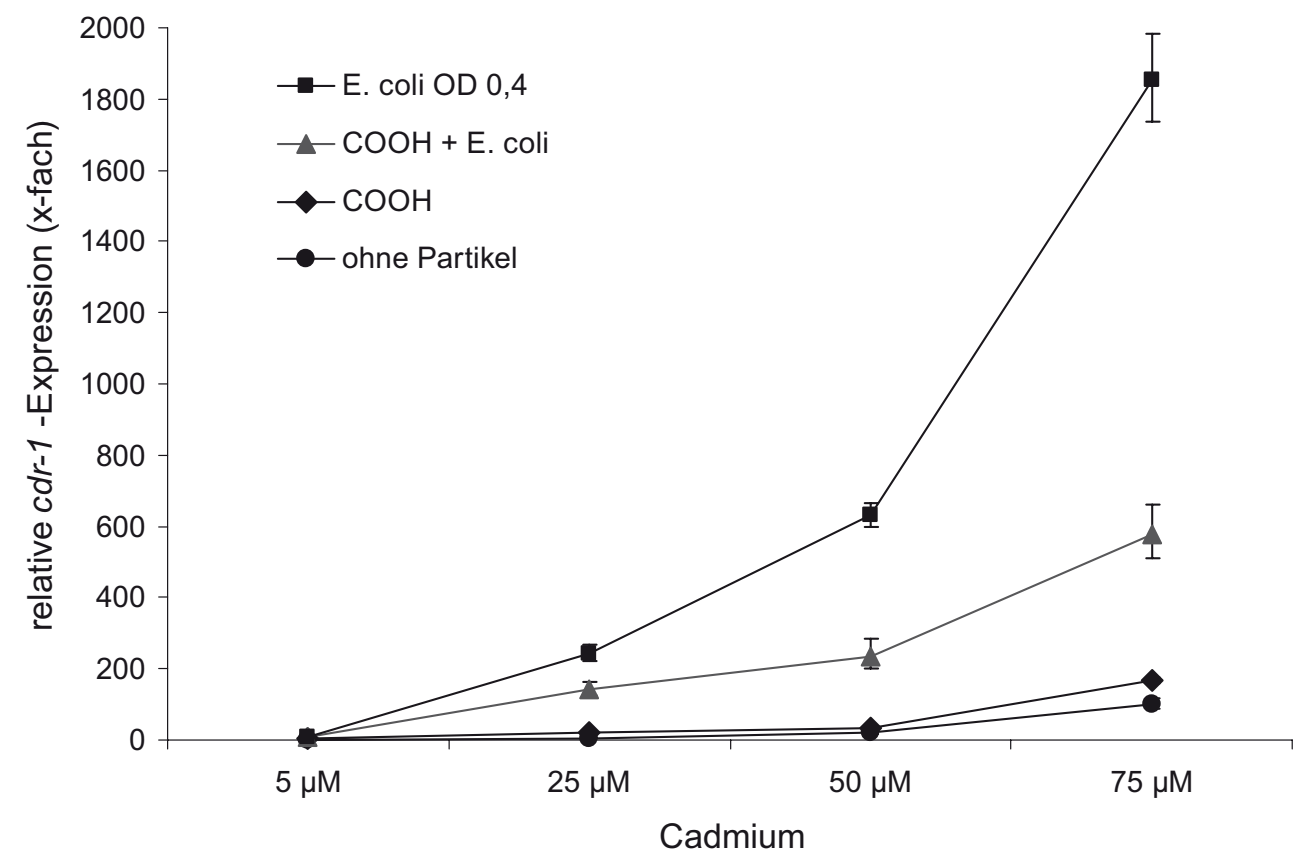


des Gens hatte ein Maximum nach $6 \mathrm{~h}$ bei einer Exposition über das Flüssigmedium, in Anwesenheit von E. coli als Nahrungspartikel war das Induktionsmaximum bereits nach $4 \mathrm{~h}$ erreicht (Abb. 2). Die Exposition über Bakterien verstärkte nicht nur die cyp-35C1-Expression, sondern auch die negativen Effekte auf die Reproduktion. Die Flu-Anreicherung dagegen wurde bei dieser Expositionsart verringert. Die interne Verfügbarkeit ist somit wichtiger für die Erzeugung irreversibler Wirkungen als die Gesamtbelastung, was auf einen spezifischen Wirkmechanismus hinweist.

\section{Ausblick}

Durch die vorgelegten Untersuchungen wurde gezeigt, dass für benthische Organismen, die sich von Bakterien ernähren, eine Schadstoffexposition über Nahrungspartikel entscheidend für toxische Wirkungen sein kann. Eine Risikoanalyse und eine Entwicklung von Qualitätskriterien für Sedimente und Biota muss daher die Exposition über Partikel allgemein und speziell über Nahrungspartikel berücksichtigen.

\section{Literatur}

Di Toro DM, McGrath JA, Hansen DJ, Berry WJ, Paquin PR, Mathew $\mathrm{R}$ et al. (2005) Predicting sediment metal toxicity using a sediment biotic ligand model, Methodology and initial application. Environ Toxicol Chem 24:2410-2427

Matthäi A (2009) Zur Bedeutung der Schadstoffexposition auf die Genexpression und Reproduktion von Caenorhabditis elegans. Dissertation der TUHH, Hamburg, $88 \mathrm{~S}$

MERAG (2007) Incorporation of Bioavailability for Water, Soils and Sediments. Metals Risk Assessment Guidance (MERAG) Fact Sheet 05. ICMM, London, 23 pp, http://www.euras.be/assets/files/MERAG/MERAG\%20FS\%2005\%20Jan\%2007.pdf, 12.05.2009

Offermann K, Matthäi A, Ahlf W (2009) Assessing the importance of dietborne cadmium and particle characteristics on bioavailability and bioaccumulation in the nematode Caenorhabditis elegans. Environ Toxicol Chem. DOI 10.1897/08-272.1 